\title{
Aptidão física de escolares com deficiência intelectual leve
} Physical fitness of students with mild intellectual disabilities

Maria Denise Justo Panda', Marilia Basilio Puglia'

'Universidade de Cruz Alta (Unicruz), Cruz Alta, RS, Brasil.

Recebido em: 14/06/2016 / Aceito em: 30/06/2016

dpanda@ibest.com.br

\section{RESUMO}

Atualmente, visualizamos muitas escolas com um trabalho significativo e contributivo para o processo de inclusão, com esforços direcionados para o desenvolvimento integral dos alunos com necessidades educacionais especiais nas escolas regulares e a educação física tem um papel importante nesse processo. Objetivo: avaliar a aptidão física relacionada à saúde e ao desempenho esportivo dos escolares diagnosticados com deficiência Intelectual leve, de três escolas participantes do Programa Institucional de Bolsa de Iniciação à Docência - PIBID/UNICRUZ/Educação Física. Método: foram sujeitos do estudo 21 escolares, com idades entre 7 e 15 anos. $O$ instrumento utilizado foi o Manual de testes e avaliação PROESP-BR (Projeto Esporte Brasil). Resultados: as variáveis que avaliaram a saúde dos educandos desencadearam maior preocupação, pois mostraram que a maioria dos alunos das três escolas está na zona de risco, principalmente no IMC (índice de massa corporal), estando eles acima do seu peso ideal, ou seja, com sobrepeso e até casos de obesidade. Outro resultado preocupante foram os índices de flexibilidade que mostram problemas de mobilidade corporal. As variáveis relacionadas ao desempenho esportivo desses alunos não foram tão alarmantes, mas estão aquém do esperado. Considerações finais: os resultados do estudo foram socializados e ofereceram indicadores para um planejamento de atividades, de acordo com as reais necessidades dos alunos, levando em conta que as oficinas de educação psicomotora do PIBID/UNICRUZ/ Educação Física devem apresentar significância para todos os seus participantes.

Palavras-chave: Aptidão Física; Escolares; Deficiência Intelectual.

\section{ABSTRACT}

Currently we envision many schools with significant work and contributing to the process of inclusion, with efforts targeted to the comprehensive development of students with special educational needs in regular schools, and physical education has an important role in this process. Objective: evaluate the physical fitness and health and sports performance of diagnosed children with mild intellectual disabilities three schools participating in the Institutional Program of Initiation Scholarship to Teaching - PIBID/UNICRUZ/ Physical Education. Method: they were subjects of the 21 school study, aged 7 to 15 years. The instrument used was the manual testing and evaluation PROESP-BR (Project Sport Brazil). Results: the variables which assessed the health of students sparked greater concern because they showed that most students of the three schools are in the risk zone mainly in BMI (body mass index) while they are above their ideal weight, ie, overweight and even obesity cases. Another worrying result was the flexibility indexes showing body mobility issues. The variables related to the sports performance of these students were not so alarming, but are less than expected. Closing remarks: the study results were socialized and offered indicators for planning activities according to the real needs of students, taking into account that the psychomotor education workshops PIBID/UNICRUZ/Physical Education must have significance for all participants.

Keywords: Physical Fitness; Schoolchildren; Intellectual Disabilities. 


\section{INTRODUÇÃO}

O processo inclusivo vem evoluindo, desde a década de 70, momento em que foi necessário que as crianças com necessidades educacionais especiais fizessem parte da escola. A partir de então, criam-se escolas segregadas, onde os alunos com necessidades educacionais especiais eram colocados em escolas especializadas. Os egressos das escolas segregadas mostraram que a prática desenvolvida não atendeu às necessidades desses alunos, pois havia como ideia principal, a preocupação de apenas inseri-los na sala de aula. Esse método não foi eficaz devido às condições nas quais essa escola foi estruturada, tornando-se somente um depósito de alunos com necessidades especiais. Nos anos 80, a educação integradora veio tentar superar a segregação, buscando como alternativa a inclusão desses alunos nas escolas regulares. Importante ressaltar que, a integração como filosofia educacional abarca a aceitação de acomodamentos específicos para modificar sistemas de ensino, sistema de avaliação, sistema de classificação, programas e atividades, etc. ${ }^{1}$

Porém, essa iniciativa encontrou muita dificuldade para ser efetivada pela escola, que apenas recebeu este alunado, fazendo com que os professores tivessem que se adaptar à nova realidade, sem o devido preparo. Esse processo de inclusão, inicialmente, contribuiu para que a exclusão acontecesse, ao contrário do que se pretendia. Com o passar do tempo, a escola foi buscando recursos, adaptando a estrutura física, buscando e formando continuamente professores, interagindo com a família e, atualmente visualizamos muitas escolas com um trabalho contributivo para o processo de inclusão. Esses esforços estão direcionados ao desenvolvimento integral dos alunos com necessidades especiais nas escolas regulares, sendo que as práticas pedagógicas devem contribuir para o seu desenvolvimento cognitivo, afetivo, social e motor.

Levando em conta que a prática de atividade física é fundamental para o desenvolvimento humano em todas as fases da vida, em especial na fase escolar, é que as aulas de Educação Física se tornam extremamente importantes para o aluno com necessidades especiais, pois as atividades físicas, quando adaptadas às necessidades desses alunos, contribuem para seu aprendizado, tanto motor, quanto cognitivo. ${ }^{2}$ Todos os alunos devem participar da Educação Física e é o docente o principal responsável para que isto aconteça, uma vez que cabe a ele elaborar seus planos de aula de maneira que estejam adaptados às práticas de todos os alunos. Em todas as atividades é necessário respeitar às limitações das pessoas com deficiência, tendo o cuidado de realizar de maneira progressiva e regular. ${ }^{3}$

Entre as necessidades educacionais especiais está a deficiência intelectual, que pode ser originada na fase pré-natal, perinatal ou pós-natal. Pode ser resultante de causa genética ou de determinadas situações, como: baixa oxigenação cerebral no parto; uso de álcool ou drogas, por parte da mãe: baixo peso; pobreza; falta de instrução e falta de acompanhamento pré-natal. É comum que as crianças com deficiência intelectual apresentem inúmeras dificuldades motoras, pois, dependendo do que ocasionou essa deficiência, poderá comprometer várias áreas, como: organização espacial, lateralidade, além da área cognitiva. O baixo desempenho motor relaciona-se à falta de atenção e compreensão, pois o aluno não consegue manter a atenção no que the é solicitado, dificultando o entendimento e afetando a aprendizagem. ${ }^{4}$

A disciplina Educação Física faz parte do currículo da escola e não pode ficar indiferente ou neutra em relação ao movimento de inclusão. ${ }^{3,5}$ Ela deve constituir-se como "adjuvante" do processo de inclusão escolar e social. ${ }^{5}$ Assim como nas outras disciplinas, na Educação Física também não existe um método ideal para lidar com a inclusão, porém cabe ao professor lançar métodos e propostas que eliminem as barreiras e que proporcione aos seus alunos com deficiência, diferentes aprendizagens. $^{3}$

A fim de que aconteça a inclusão nas aulas de Educação Física, não basta adaptar a disciplina para que o aluno com deficiência intelectual possa participar, é preciso algo mais. Para tanto, é importante que o professor adote objetivos, conteúdos e métodos que considerem a diversidade humana e que contribuam para a construção de uma sociedade inclusiva e não somente uma aula inclusiva. O alvo principal é a mudança no modo de pensar e ver de toda a sociedade escolar e não só a participação dos alunos em aulas, nas escolas regulares; vai muito além, refere-se à mudança de ideias. Com a inclusão, o desejo é transformar a sociedade em um local onde se saiba conviver e respeitar as diferenças.

A inclusão faz parte da proposta pedagógica de diversas escolas, na cidade de Cruz Alta/RS/Brasil, e entre essas escolas estão as três que integram o PIBID - Programa Institucional de Bolsa de Iniciação à Docência, na subárea Educação Física. Neste programa, os acadêmicos do curso de Educação Física desenvolvem oficinas de educação psicomotora, três vezes por semana, com duração de 50 minutos. A abordagem metodológica segue a linha desenvolvimentista e o os estudos científicos do programa voltaram-se, inicialmente, à avaliação motora, no entanto, esses mesmos estudos indicaram a necessidade de envolver a questão da aptidão física.

As crianças que se encontram na faixa etária do Ensino Fundamental devem realizar, pelo menos, 30 a 60 minutos de atividades físicas diárias, sendo variadas e de acordo com a idade e a base desenvolvimentista, para o desenvolvimento da aptidão física, nos seus diversos componentes. A aptidão física pode ser entendida como um conjunto de atributos relacionados à capacidade de executar atividade física em associação à composição genética do indivíduo, juntamente com a manutenção de nutrição adequada.

O presente estudo valoriza o processo de avaliação para detectar o nível de desenvolvimento dos alunos no sentido de obter indicadores que possam nortear um planejamento de atividades de acordo com a realidade dos alunos. Considerando que as aulas de Educação Física devem apresentar significância para todos os seus participantes, avaliar a aptidão física, tanto relacionada à saúde, como ao desempenho esportivo dos escolares com necessidades educacionais especiais, participantes do PIBID/ UNICRUZ/ Educação Física, serve para conhecer as características desses alunos, suas necessidades e possibilidades. 
Este estudo detectou, inicialmente, os alunos com deficiência intelectual leve do referido programa e teve como objetivo avaliar os componentes da aptidão física relacionada à saúde e ao desempenho esportivo desses alunos. A intenção remete ao conhecimento da situação dos componentes da aptidão física para a saúde, se estão na zona de risco ou na zona saudável, bem como detectar se existem alunos com possibilidades de desempenho esportivo, para que sejam encaminhados ao grupo de treinamento paraolímpico da cidade.

A realização desse estudo teve como objetivo identificar quais são os maiores déficits desses alunos e as áreas mais debilitadas, para que, assim, os professores realizem ações na busca de uma melhor aptidão física.

\section{MÉTODO}

Estudo de campo, do tipo descritivo, de caráter diagnóstico. A população foi constituída por todos os alunos das três escolas que participam, regularmente, das oficinas de educação psicomotora do PIBID/ UNICRUZ/Educação Física, num total de seiscentos e trinta e três (633) escolares. As escolas possuem um professor responsável pelo atendimento aos alunos com necessidades educacionais especiais em uma sala de recurso. Nesta sala, cada aluno possui um histórico individual e, conforme contato com o professor responsável pelo Atendimento Educacional Especializado - AEE foram obtidos os laudos dos alunos com diagnóstico de deficiência intelectual leve, formando a amostra do estudo do tipo intencional, num total de vinte e um (21) escolares, sendo oito (08) do sexo feminino e treze (13) do sexo masculino, com idades entre sete (07) e quinze (15) anos, sendo três (03) do segundo ano, quatro (04) do terceiro ano, nove (09) do quarto ano e cinco (05) do quinto ano.

Inicialmente, foi realizado contato com a coordenação institucional do PIBID/UNICRUZ para solicitar autorização para a realização do estudo. Após foi realizada a comunicação com as escolas participantes do programa, fazendo contato com os professores e a direção para explicar os objetivos e os procedimentos do estudo. Em seguida, foi explicado aos alunos e seus pais os objetivos e os procedimentos da pesquisa e solicitado que voluntariamente assinassem o Termo de Consentimento e Assentimento Livre Esclarecido.

$O$ instrumento utilizado foi o Manual de testes e avaliação PROESP-BR-Projeto Esporte Brasil, que avalia a aptidão física relacionada à saúde e ao desempenho esportivo. ${ }^{7}$ Os resultados foram organizados em uma planilha que seguiu os parâmetros de classificação do PROESP-BR - Projeto Esporte Brasil. ${ }^{7}$

Este estudo foi submetido ao Comitê de Ética em Pesquisa da UNICRUZ e aprovado, sob o parecer $\mathrm{n}^{\circ}$ 1.234.094, de 20/11/2015, conforme especifica a Resolução 446, de 12/12/2012.

\section{RESULTADOS E DISCUSSÃO}

Os resultados seguem apresentados, na forma de tabela, de acordo com os objetivos do estudo. Inicialmente, a pesquisa mensurou e qualificou as variáveis da aptidão física relacionada à saúde: Índice de massa corporal (IMC), aptidão cardiorrespiratória (ACR), flexibilidade (FLEX) e resistência muscular localizada (RML). Após a aplicação dos testes, o estudo seguiu o conjunto de critérios e normas de avaliação do PROESP-BR Programa Esporte Brasil, ${ }^{7}$ classificando os escolares em ZS - Zona Saudável e ZR - Zona de Risco para a Saúde.

Conforme os resultados visualizados na tabela 1, os alunos da Escola $A(n=08)$ apresentam resultados que, mostram que a maioria se encontra na zona de risco para a saúde, em todas as variáveis, IMC (07 alunos), ACR (06 alunos), FLEX (07 alunos), RML (05 alunos). Com relação à Escola $B(n=05)$, percebeu-se que, na maioria das variáveis avaliadas, IMC (04 alunos), ACR (04 alunos), FLEX (04 alunos), os alunos encontravam-se na zona de risco à saúde, sendo apenas na variável RML (04 alunos), que os alunos estão na zona saudável. $\mathrm{Na}$ escola $C(n=08)$, também foi observado que, na maioria das variáveis, os alunos também se encontram na zona de risco à saúde, ACR (05 alunos), FLEX 105 alunos), RML (07 alunos). Já, na variável IMC, metade dos alunos se encontra na zona de risco à saúde, o que é preocupante e leva a perceber a necessidade de realizar ações na escola que tratem desses aspectos relacionados à saúde dos educandos.

Um estudo realizado no município de Ponta Grossa/PR encontrou resultados semelhantes ao encontrado neste, também realizado com alunos de uma escola pública que teve como objetivo avaliar os níveis de aptidão física relacionada à saúde de 104 crianças do ensino fundamental (47 do sexo masculino e 57 do sexo feminino) de 8 a 10 anos e que utilizou o mesmo protocolo de testes PROESP-BR. O referido estudo apresentou diferenças entre os sexos, nos seguintes testes: flexibilidade, na faixa etária de oito anos; abdominal, nos grupos de oito e nove anos; e no teste de 9 minutos, no grupo de 10 anos. Observou-se que o grupo feminino apresentou maiores características de sobrepeso,

Tabela 1 - Aptidão física para a saúde dos escolares avaliados.

\begin{tabular}{|c|c|c|c|c|}
\hline & & Escola A $(n=08)$ & Escola B $(n=05)$ & Escola C $(n=08)$ \\
\hline \multirow[t]{2}{*}{ IMC } & ZS & 01 & 01 & 04 \\
\hline & ZR & 07 & 04 & 04 \\
\hline \multirow[t]{2}{*}{ ACR } & ZS & 02 & 01 & 03 \\
\hline & ZR & 06 & 04 & 05 \\
\hline \multirow[t]{2}{*}{ FLEX } & ZS & 01 & 01 & 03 \\
\hline & ZR & 07 & 04 & 05 \\
\hline \multirow[t]{2}{*}{ RIML } & ZS & 03 & 04 & 01 \\
\hline & ZR & 05 & 01 & 07 \\
\hline
\end{tabular}

Legenda: IMC - Índice de massa corporal; ACR - Aptidão cardiorrespiratória; FLEX - Flexibilidade; RML - Resistência muscular localizada; ZS - Zona saudável; ZR - Zona de risco. 
quando comparado ao masculino. Já, entre as idades não foram verificadas diferenças estatisticamente significativas. Os resultados observados não apresentaram níveis satisfatórios de aptidão física relacionada à saúde, revelando um risco aumentado de doenças crônicas não transmissíveis aos escolares. ${ }^{8}$

Em uma pesquisa com características semelhantes a esta, realizada com alunos de um PIBID, no qual também foi usado o mesmo instrumento, ficou constada que a avaliação da aptidão física em jovens consiste em uma importante alternativa disponível aos professores de Educação Física, a fim de avaliar e monitorar o desempenho dos seus alunos e teve, nos resultados dos testes relativos ao estado nutricional dos alunos, um evidente percentual de sobrepeso e casos de obesidade, sendo que as variáveis relacionadas à aptidão física para a saúde mostrou um baixo nível de classificação dos alunos. ${ }^{9}$

Outro estudo realizado com crianças que possuem deficiência intelectual chegou à conclusão de que poucas são as informações científicas que trazem dados fisiológicos da resposta que indivíduos deficientes mentais com e sem Síndrome de Down apresentam ao serem submetidos à prática de exercícios. O objetivo da pesquisa foi avaliar os níveis de aptidão física, antropometria e composição corporal de indivíduos deficientes mentais do sexo masculino, com e sem Síndrome de Down. Nos testes de avaliação antropométrica, foram coletadas informações sobre IMC (peso e estatura). Os dados coletados que avaliaram a aptidão física basearam-se na bateria de testes da Rede CENESP que utiliza o PROESP/BR. Foi possível concluir que alunos que não possuíam Síndrome de Down apresentaram resultados superiores aos indivíduos com síndrome de Down, em todos os testes, tais como: aptidão física, antropometria e composição corporal. A aceitação deu-se dentro dos índices propostos para os indivíduos deficientes com Síndrome de Down. O objetivo de comparar o nível de aptidão física em indivíduos deficientes com e sem síndrome de Down foi alcançado. Pode-se verificar, com relação ao estado nutricional, que o IMC dos grupos pesquisados se apresentou superior ao proposto pela literatura. ${ }^{10}$

Um estudo realizado com indivíduos com deficiência mental da APAE de Campo Grande/MS, com idade entre 8 a 17 anos, que também teve como objetivo avaliar a aptidão física relacionada à saúde e como instrumento os testes do PROESP-BR, utilizou uma classificação através de três critérios, abaixo da zona saudável, zona saudável e acima da zona saudável. Os resultados evidenciaram que, na variável IMC, 55,8\% dos indivíduos analisados estavam acima da zona risco e $44,4 \%$ estavam acima ou abaixo dos níveis esperados, sendo $25,6 \%$ com excesso de peso e $18,6 \%$ com peso abaixo do indicado. Já, na variável flexibilidade, $44,19 \%$ dos avaliados estavam abaixo da zona saudável, 26,75\% na zona saudável e 29,06\% acima da zona saudável. A variável RML mostrou um resultado bem alarmante e mostrou o que menciona na literatura, que esses indivíduos têm níveis de força e resistência muscular baixo, sendo 93\% abaixo da zona saudável, $3,5 \%$ na zona saudável e 3,5\% acima da zona saudável. A resistência aeróbica apresentou dados mais preocupantes, com 97,5\% abaixo da zona saudável, tendo apenas $2,5 \%$ na zona saudável e nenhum acima da zona saudável. Pode-se constatar que são necessários mais estudos relacionados à aptidão física para a saúde, com o intuito de avaliar essa população que apresentou níveis bem deficitários. A maior dificuldade foi comparar os resultados com estudos semelhantes, pois são poucas pesquisas na área da avaliação física relacionada a deficientes intelectuais. ${ }^{11}$

A seguir, apresenta-se a mensuração e conceituação das variáveis da aptidão física relacionada ao desempenho esportivo, que são: força explosiva de membros superiores; força explosiva de membros inferiores; agilidade e velocidade. Após a aplicação dos testes citados, o estudo seguiu o conjunto de critérios e normas de avaliação do PROESP-BR - Programa Esporte Brasil. ${ }^{7}$ Os resultados podem ser visualizados na tabela a seguir:

Ao analisar a tabela 2, com as variáveis que possibilitariam indicar alunos com desempenho esportivo, que pudessem ser conduzidos para as equipes escolares, os resultados mostram que as escolas não possuem nenhum aluno com potencial atlético. Apenas na Escola A que 04 alunos obtiveram classificação Excelente em Força de Membros Inferiores, 01 aluno Excelente em Velocidade, quatro alunos Muito Bom em Agilidade, 01 aluno Muito Bom em Velocidade e 01 aluno Muito Bom em Força de Membros Inferiores. Um desses alunos poderá vir a ser destaque desde que seja trabalhada a variável Força de Membros Superiores, considerando que ele foi Excelente e Muito Bom nas outras variáveis

Tabela 2 - Aptidão física para o desempenho esportivo dos escolares avaliados.

\begin{tabular}{|c|c|c|c|c|c|c|}
\hline & Teste & Excelente & Muito Bom & Bom & Razoável & Fraco \\
\hline Escola A & Força MMSS & 0 & 0 & 0 & 0 & 08 \\
\hline \multirow[t]{3}{*}{$n=08$} & Força MMII & 04 & 01 & 02 & 0 & 01 \\
\hline & Agilidade & 0 & 04 & 01 & 01 & 02 \\
\hline & Velocidade & 01 & 01 & 0 & 0 & 06 \\
\hline Escola B & Força MMSS & 0 & 02 & 0 & 0 & 03 \\
\hline \multirow[t]{3}{*}{$n=05$} & Força MMII & 0 & 0 & 0 & 0 & 05 \\
\hline & Agilidade & 0 & 0 & 0 & 0 & 05 \\
\hline & Velocidade & 0 & 01 & 0 & 0 & 04 \\
\hline Escola C & Força MMSS & 0 & 01 & 02 & 02 & 03 \\
\hline \multirow[t]{3}{*}{$n=08$} & Força MMII & 0 & 02 & 01 & 03 & 02 \\
\hline & Agilidade & 0 & 0 & 05 & 02 & 01 \\
\hline & Velocidade & 0 & 0 & 03 & 04 & 01 \\
\hline
\end{tabular}

Legenda: Força MMSS - Força de membros superiores; Força MMII - Força de membros inferiores. 


\section{do Desempenho Esportivo.}

Uma pesquisa com o mesmo propósito de avaliar os indicadores da aptidão física referente à saúde e ao desempenho esportivo de escolares utilizou uma bateria de cinco testes motores, salto em distância parado (Força de MMII), arremesso de "medicineball", barra modificada (Força de MMSS), corrida de 20 metros (velocidade) e quadrado (agilidade). Nessa pesquisa, participaram 526 alunos entre 7 e 15 anos, do Ensino Fundamental da cidade de Rio Grande/RS. Os resultados apontam que o desempenho em todos os testes foi superior para os rapazes e aumentou diretamente com a faixa etária. Estes foram os principais preditores da aptidão física, dentre as variáveis examinadas. O tipo de escola (pública/particular) só teve influência sobre o desempenho, no teste de arremesso de "medicineball", em que alunos da rede privada obtiveram valores médios maiores. Escolares da zona urbana atingiram um melhor desempenho em todos os testes, exceto no teste de barra modificada (sem diferença por região geográfica). A realização de pesquisas com o mesmo enfoque em outros locais do Brasil permitirá explorar a consistência destes achados e compreender possíveis diferenças. ${ }^{12}$

Um estudo com características semelhantes a este, realizado com alunos de um PIBID, no qual também foi usado o mesmo instrumento, entendeu que a avaliação da aptidão física em jovens fornece informações que servirão como indicadores para o planejamento de atividades mais adequadas. $O$ estudo teve como objetivo avaliar a aptidão física relacionada à saúde e ao desempenho esportivo de escolares, sendo que participaram 162 alunos, na faixa etária de 10 a 12 anos. A aptidão física para o desempenho esportivo determinou melhores resultados, porém não foi encontrado nenhum aluno com índices suficientes que possam indicar a manifestação de um talento esportivo. Concluiu-se que as variáveis da aptidão física para a saúde precisam de mais atenção, pois foram as que se apresentaram com os piores índices e são as mais importantes para o bem-estar dos alunos ${ }^{9}$, o que se assemelha aos resultados encontrados no presente estudo.

\section{CONSIDERACִÕES FINAIS}

O presente estudo teve como objetivo identificar os alunos com deficiência intelectual leve, participantes do programa PIBID/UNICRUZ, subprojeto de Educação Física, bem como avaliar a aptidão física para a saúde e desempenho esportivo desses escolares.

Foram encontrados resultados alarmantes, principalmente com relação às variáveis referentes à saúde. No IMC (Índice de Massa Corporal), em duas das três escolas, mais de $80 \%$ dos alunos encontravam-se na zona de risco para a saúde, estando acima do seu peso ideal, ou seja, com sobrepeso e até casos de obesidade. Esses valores críticos preocupam, pois estão seriamente relacionados com a saúde desses alunos, indicando que esses educandos não têm uma alimentação adequada e uma boa nutrição para a regulação do peso ideal, podendo acarretar problemas futuros mais sérios que conduzem a doenças crônico-degenerativas.

Outro resultado que também chamou a atenção foi com relação à flexibilidade, sendo que, nas três escolas, a maioria dos alunos apresentou baixo nível de classificação, mostrando que cada vez mais as crianças e adolescentes se tornam sedentários com dificuldades de mobilidade corporal, o que leva a apresentarem bastante dificuldade, no momento da realização de atividades que utilizem outras valências físicas. 0 mesmo pode ser visualizado na variável de resistência muscular localizada, na qual os baixos índices podem se justificar pela ineficiência das atividades físicas realizadas por estes alunos e, em muitos casos, a não participação nas aulas.

As variáveis relacionadas ao desempenho esportivo também apresentaram resultados preocupantes. $\mathrm{Na}$ força de membros superiores, força de membros inferiores, agilidade e velocidade, mais de $80 \%$ dos alunos foram classificados com desempenho fraco, o que sugere aos bolsistas do Programa Institucional de Bolsa de Iniciação à Docência - PIBID/UNICRUZ/ Educação Física que trabalham com estes alunos nas três escolas a realizar uma revisão nas propostas de trabalho. Nesse sentido, enfatizar essas valências físicas e não só voltar o trabalho apenas para o desenvolvimento motor dos alunos, bem como fortalecer a questão da integração dos alunos em todas as atividades das oficinas de educação psicomotora, buscando a participação efetiva também dos que apresentam deficiência intelectual leve.

Comparando os resultados obtidos, nesse estudo, com outras pesquisas, porém com alunos que não apresentam deficiência intelectual, que estão na normalidade, foram encontrados resultados bem semelhantes.

Portanto, com a realização deste estudo pôde-se identificar quais foram as áreas mais deficitárias, ou seja, quais necessitam mais atenção e um trabalho mais específico nas oficinas de educação psicomotora, com atividades que promovam a melhoria da aptidão física, tanto para a saúde quanto para o desempenho esportivo desses alunos caracterizados com deficiência intelectual leve, buscando com isso uma melhora no condicionamento físico e, principalmente, na saúde e no bem-estar dos educandos, o que favorece, também, outras aprendizagens.

Os resultados deste estudo foram socializados em uma reunião pedagógica dos coordenadores, supervisores e bolsistas do PIBID/UNICRUZ/Educação Física.

\section{AGRADECIMENTOS}

PIBIC/UNICRUZ - Programa Institucional de Bolsa de Iniciação Científica com fomento da Universidade de Cruz Alta.

PIBID/CAPES - Programa Institucional de Bolsa de Iniciação à Docência com fomento do Coordenação de Aperfeiçoamento de Pessoal de Ensino Superior.

\section{REFERÊNCIAS}

1. Fonseca V. Educação Especial. 2. ed. Porto Alegre- RS: Artes Médicas, 1987. 127p.

2. Oliveira AAB, Tatsh T, Panda MDJ, Batistella PA, Rossato VM. A questão da inclusão dos alunos com deficiência mental na escola regular e as aulas de Educação Física. Anais do XVIII Seminário Internacional de Educação do Mercosul. UNICRUZ. Cruz Alta/RS, 2013. 
3. Cidade RE, Freitas PS. Educação Física e Inclusão: Considerações para a Prática Pedagógica na Escola. Brasília: Ministério da Educação. Revista Integração. 2002; 14 (Edição Especial - Educação Física Adaptada): 27-30.

4. Krebs P. Retardo Mental. In: WINNICK, Joseph P. Educação Física e Especial. Barueri-SP: Manole. 2004. p.125-143.

5. Aguiar JS, Duarte E. Educação inclusiva: um estudo na área da educação física. Revista Brasileira de Educação Especial 2005;11(2):223-240.

6. GallahueDL, DonnellyFC. EducaçãoFísica Desenvolvimentista para todas as crianças/ 4 ed. São Paulo: Phorte, 2008. 726p.

7. Gaya A, Lemos A, Gaya A, Teixeira D, Pinheiro E, Moreira R. PROESP-Br. Projeto Esporte Brasil /. Manual de Testes e Avaliação. 2015. (https://www.ufrgs.br/proesp/arquivos/ manual-proesp-br-2015.pdf )

8. Fonseca HAR. Aptidão Física à saúde de escolares da escola pública de tempo integral. Acta Scientiarum. Health Sciences 2010;32(2):155-161.

9. Furhmann M, Panda, MDJ. Aptidão Física relacionada à saúde e ao desempenho esportivo de escolares do PIBID/UNICRUZ/ Educação Física. Revista Biomotriz 2015;9(1):81-99.

10. Santos JR, Oliveira TA, Leão AS. Variáveis da aptidão física de indivíduos deficientes mentais com ou sem síndrome de down - EfDeportes. Revista Digital Buenos Aires 2010;15(149):78-89.

11. Bacciotti SM. Avaliação da aptidão física relacionada à saúde em indivíduos de 8 a 17 anos com deficiência mental da APAE de Campo Grande- MS. 89 f. 2007. Dissertação - Mestrado em Ciências da Saúde. Universidade de Brasília. 2007. 89p.

12. Dumith SC. Aptidão física relacionada ao desempenho motor em escolares de sete a 15 anos - Revista Brasileira de Educação Física e Esporte 2010;24(1):5-14.

Como citar: PANDA, Maria Denise Justo; PUGLIA, Marilia Basilio. Aptidão física de escolares com deficiência intetectual leve. Cinergis, Santa Cruz do Sul, v. 17, n. 2, jun. 2016. ISSN 2177-4005. Disponível em: <https://online.unisc.br/seer/index.php/ cinergis/article/view/7723>. Acesso em: 04 jul. 2016. doi:http://dx.doi.org/10.17058/cinergis.v17i2. 7723. 